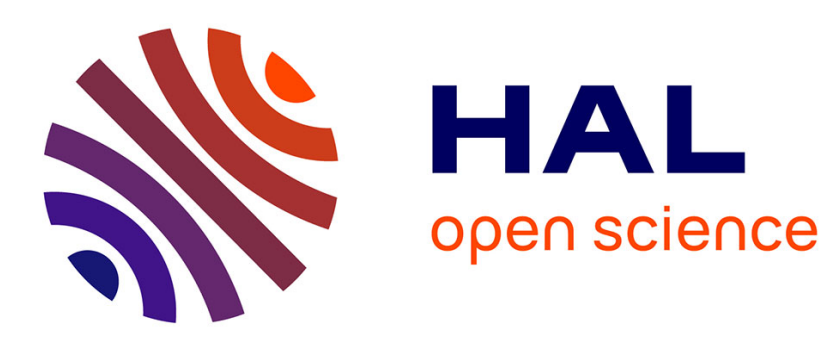

\title{
Trajectory Planning of Kinematically Redundant Parallel Manipulators by Using Multiple Working Modes
}

\author{
Daniel Reveles Ramos, J. Alfonso Pamanes, Philippe Wenger
}

\section{To cite this version:}

Daniel Reveles Ramos, J. Alfonso Pamanes, Philippe Wenger. Trajectory Planning of Kinematically Redundant Parallel Manipulators by Using Multiple Working Modes. Mechanism and Machine Theory, 2016, 98, pp.216-230. 10.1016/j.mechmachtheory.2015.09.011 . hal-01712832

\section{HAL Id: hal-01712832 \\ https://hal.science/hal-01712832}

Submitted on 19 Feb 2018

HAL is a multi-disciplinary open access archive for the deposit and dissemination of scientific research documents, whether they are published or not. The documents may come from teaching and research institutions in France or abroad, or from public or private research centers.
L'archive ouverte pluridisciplinaire HAL, est destinée au dépôt et à la diffusion de documents scientifiques de niveau recherche, publiés ou non, émanant des établissements d'enseignement et de recherche français ou étrangers, des laboratoires publics ou privés. 


\title{
Trajectory Planning of Kinematically Redundant Parallel Manipulators by Using Multiple Working Modes
}

\author{
Daniel Reveles R. ${ }^{\mathrm{a}}$, J. Alfonso Pamanes G. ${ }^{\mathrm{a}}$, Philippe Wenger ${ }^{\mathrm{b}}$ \\ ${ }^{a}$ Instituto Tecnológico de la Laguna (ITLag), Blvd. Revolución y Cuauhtémoc, Torreón, Coah. Mexico, CP 27000 \\ ${ }^{b}$ Institut de Recherche en Communications et Cybernétique de Nantes (IRCCyN), 1 Rue de la Noë, BP 92101, 44321 Nantes Cedex \\ 03, France
}

\begin{abstract}
A method for joint trajectory planning of kinematically redundant parallel manipulators is presented in this paper. The method is based on the use of feasibility maps that allow the graphical assessment of postures related to the different working modes of the manipulator. Thus, in such maps suitable trajectories are found in order to improve the performance of the manipulator and to avoid parallel singularities. When the desired path cannot be achieved by using only one working mode of the manipulator without crossing parallel singularities then, by applying the approach proposed here, a number of working modes could be chosen and combined to complete the whole path. The method can be applied to parallel manipulators with one degree of kinematic redundancy, whose legs allow the changing of working modes. The efficiency of this method is shown in a case study with a 3-RRR parallel manipulator (note that this manipulator becomes kinematically redundant if the orientation of the mobile platform is not specified for a desired task), in which postures associated to four working modes are employed in such a way that parallel singularities can be avoided. The obtained joint trajectories are applied to an experimental prototype to successfully execute the desired task.
\end{abstract}

Keywords: Parallel manipulators, kinematic redundancy, trajectory planning, working modes, singularity avoidance.

\section{Introduction}

The singularities associated with the direct kinematics of parallel manipulators (referred to as "parallel singularities" in this paper) may cause serious losses of stiffness and even control when they are approached [1]. Avoiding such singularities during motion has then drawn the interest of many researchers [2-7]. For non-redundant parallel manipulators, several strategies have been proposed to guaranty that the path to be followed by the moving platform remains sufficiently far from singular configurations [8-9]. Abdellatif and Heiman [10] synthesize paths in the 3D space by avoiding both singularities and collisions. In the aforementioned methods for path-planning it is assumed that only one working mode can be used to accomplish the desired task.

When a non-redundant parallel manipulator has several working modes (associated with the different inverse kinematics solutions), an appropriate selection of the working mode may help avoiding a parallel singularity [11-12]. A strategy was proposed by Figielski et al. [13] for trajectory planning of a five-bar mechanism by changing the working mode if necessary during pick-and-place motions. The authors applied the active-joint space to define the possible trajectories that can be used to achieve the task. This method was validated by applying it for trajectory planning of the DexTAR manipulator [14]. On the other hand, Macho et al. [15] developed an extensive analysis of the workspace and singularities of the mechanism. 
As a result, the authors proposed a strategy for path planning by changing the working mode. Later, to solve this problem, another approach based on the use of performance maps was presented in [16], which accomplishes the change of the working mode by using a posture of the manipulator far away from parallel singularities.

On the other hand, when the parallel manipulator is kinematically redundant, the issue is different as the inverse kinematic problem admits infinitely many solutions. In trajectory planning of this kind of manipulators the histories of the active joint variables must be obtained in such a way that the end-effector can describe a specified path (main task), and simultaneously the posture of the manipulator achieves a secondary task. This secondary task could be specified as the optimization of the manipulator's kinetostatic performance, the obstacle avoidance or some other assigned by the user.

The problems of optimization of kinematic performance and obstacle avoidance have been investigated in the literature for trajectory planning of serial [17-23] and parallel [24-32] kinematically redundant manipulators. In [29] the authors proposed two consistent kinetostatic performance indices, which were applied to solve the kinematic redundancy when the desired task is carried out. The postures of the manipulator during the obtained motion were kept far away from both parallel and serial singularities. In another work, Cha et al. [30] applied the redundancy to avoid parallel singularities based on local optimization. Aginaga et al. [31] proposed to use the inverse singularity (a transition between two working modes) to improve the stiffness of trajectories. Carretero at al. optimized the index referred to as normalized scaled incircle radius (NSIR) to solve the redundancy while the desired task is accomplished [32]. The maximization of the NSIR allows avoiding both parallel and serial singularities. It must be pointed out that the normalized condition number or other equivalent indices have been also used to measure the proximity of a manipulator to singular postures [33-34]. The approaches of references [29-32] are applied to follow specific trajectories of the mobile platform. However, they do not take into account changes of working modes, if required, to achieve a task.

The issue treated in this paper, namely the selection of a sequence of working modes for the execution of a prescribed path by a kinematically redundant parallel manipulator, has been studied in [35] based on simulation analyses. Latterly, during experiments with a prototype of the considered manipulator applying the results of that study, it was observed that significant shaking forces and vibrations occurred when changes of working mode were accomplished. These phenomena have not been appreciated in kinematic simulations. Thus, in order to be efficient on the real manipulator, additional conditions and analyses were developed in such a way that a more accurate platform motion can be specified during the changes of working modes. As a result, meaningful improvements to the previous approach in [35] were incorporated. An assessment of the improved method was completed with the experimental prototype and it was observed that the shaking forces and vibrations were eliminated. The supplementary analyses, the whole improved method and its application to the real prototype in a case study are presented in this paper. To the knowledge of the authors no other methods have been proposed to solve the problem studied here, in which tasks having specific trajectories of the mobile platform must be accomplished by kinematically redundant parallel manipulators using multiple working modes.

Some basic notions about kinematics of parallel manipulators are recalled in Section 2. Then, in Section 3 the concepts on the feasibility maps are considered. The proposed improved method and a case study are described in Section 4. The conclusion is presented in Section 5.

\section{Kinematics of the 3-RRR manipulator}

Even if the approach proposed in this paper is general and can be applied to other kinematically redundant parallel manipulators, the method is illustrated by applying it to a 3-RRR parallel manipulator. Thus, the analysis achieved on this manipulator can be extended to other architectures with one degree of kinematic redundancy that have multiple working modes.

A schematic representation of a 3-RRR planar parallel manipulator is shown in Figure 1. The manipulator has 3-DOF (Degrees Of Freedom); its architecture includes three RRR chains (each one named leg). Each chain connects the manipulator's base to the mobile platform in a symmetrical way. The base and the platform are triangular and equilateral, and the links lengths are the same for each leg. 


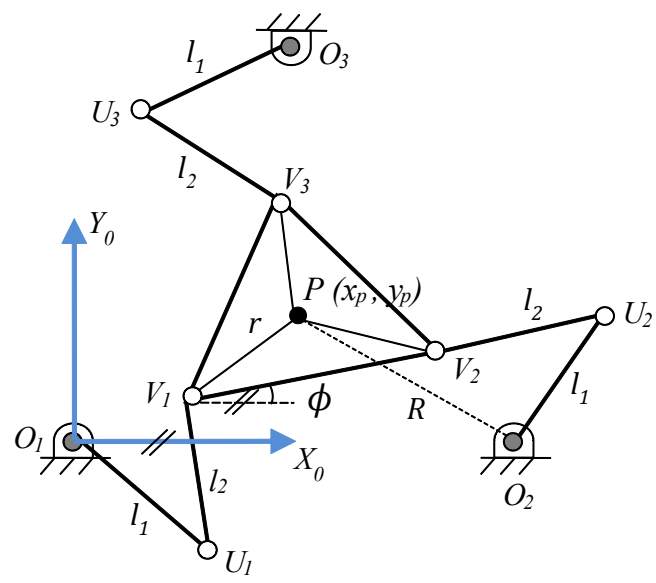

Fig. 1: Kinematic scheme of the 3-RRRR parallel manipulator.

In each chain, only the joint connecting the leg to the base is actuated. The first underlined $\mathrm{R}$ letter in the designation specifies this feature of the manipulator, and it is also graphically indicated by the gray color of joints in the kinematic scheme. The radius of the circle that passes through the vertices of the platform (resp. the base) is referred to as $r$ (resp. $R$ ). The length links are referred to as $l_{1}$ and $l_{2}$. Additionally, the joint variables of the fixed joints are the angles $\theta_{1}, \theta_{3}, \theta_{5}$, as observed in Figure 2.

To describe the position of the platform in the inertial frame $X_{0}-Y_{0}$ shown in Figure 1, the Cartesian coordinates $x_{p}, y_{p}$, of point $P$ at the centroid of the triangle are employed. The angle $\phi$ of the lower edge $\left(V_{1}-V_{2}\right)$ of the triangle with respect to the $X_{0}$ axis is used to define the platform orientation. The variables corresponding to the passive joints are $\theta_{2}, \theta_{4}$ and $\theta_{6}$. Figure 2 shows a scheme of vectors associated to the manipulator's links, which are applied to the kinematic analysis of the manipulator.

\subsection{Velocity equations and singularities}

The relationship between the vector $\mathbf{t}$ of velocities of the mobile platform and the vector $\dot{\mathbf{q}}$ of the active joint velocities can be expressed as:

$$
\mathbf{A t}=\mathbf{B} \dot{\mathbf{q}}
$$

where $\mathbf{t}=\left[\begin{array}{lll}\dot{x_{p}} & \dot{y_{p}} & \dot{\boldsymbol{\phi}}\end{array}\right]^{T}, \dot{\mathbf{q}}=\left[\begin{array}{ccc}\dot{\theta_{1}} & \dot{\theta_{3}} & \dot{\theta_{5}}\end{array}\right]^{T}$. The components of $\mathbf{t}$ are referred to the frame $X_{0}-Y_{0}-Z_{0}$, whose axes $X_{0}$ and $Y_{0}$ are those shown in Figures 1 and 2. For the considered manipulator, the matrices $\mathbf{A}$ and $\mathbf{B}$ in Equation (1) are given by:

$$
\begin{gathered}
\mathbf{A}=\left[\begin{array}{ccc}
r_{2 x} & r_{2 y} & \left(r_{2 x} r_{a y}-r_{a x} r_{2 y}\right) \\
r_{4 x} & r_{4 y} & \left(r_{4 x} r_{b y}-r_{b x} r_{4 y}\right) \\
r_{6 x} & r_{6 y} & \left(r_{6 x} r_{c y}-r_{c x} r_{6 y}\right)
\end{array}\right] \\
\mathbf{B}=\left[\begin{array}{ccc}
r_{1 x} r_{2 y}-r_{2 x} r_{1 y} & 0 & 0 \\
0 & r_{3 x} r_{4 y}-r_{4 x} r_{3 y} & 0 \\
0 & 0 & r_{5 x} r_{6 y}-r_{6 x} r_{5 y}
\end{array}\right]
\end{gathered}
$$

The matrices $\mathbf{A}$ and $\mathbf{B}$ are named, respectively, parallel and serial Jacobian matrices [1]. The terms in these matrices are defined as

$$
r_{j x}=r_{j} \cos \left(\theta_{j}\right)
$$




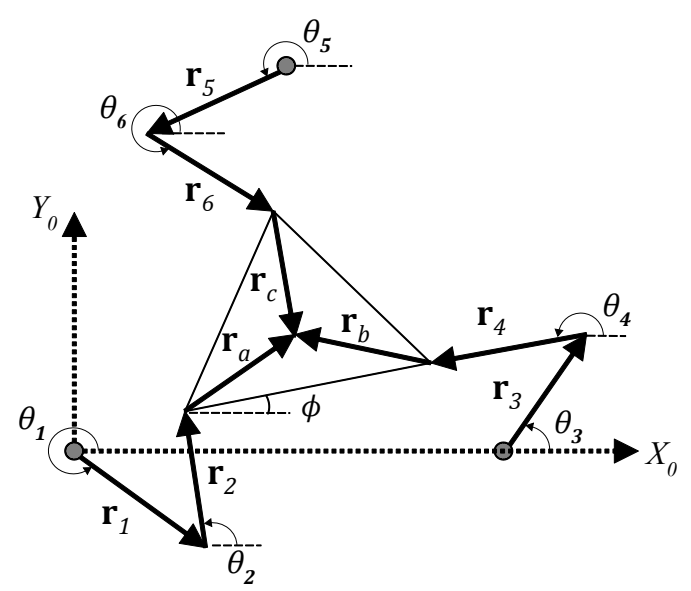

Fig. 2: Vectors associated to links of the 3-RRR parallel manipulator.

$$
r_{j y}=r_{j} \sin \left(\theta_{j}\right)
$$

where $r_{j}=\left\|\mathbf{r}_{j}\right\|$ and $r_{j}=l_{1}$ for $j=1,3,5$ and $r_{j}=l_{2}$ for $j=2,4,6 ; l_{1}$ and $l_{2}$ are the length links. The symbol $\|\cdot\|$ denotes the Euclidian norm of the argument. On the other hand, the components of vectors $\mathbf{r}_{a}, \mathbf{r}_{b}$ and $\mathbf{r}_{c}$ in the frame $X_{0}-Y_{0}$ are expressed by the following equations:

$$
\begin{gathered}
\mathbf{r}_{a}=\left[\begin{array}{c}
r_{a x} \\
r_{a y} \\
r_{a z}
\end{array}\right]=\left[\begin{array}{c}
\frac{\sqrt{3}}{2} r \cos \phi-\frac{r}{2} \sin \phi \\
\frac{\sqrt{3}}{2} r \sin \phi+\frac{r}{2} \cos \phi \\
0
\end{array}\right] \\
\mathbf{r}_{b}=\left[\begin{array}{c}
r_{b x} \\
r_{b y} \\
r_{b z}
\end{array}\right]=\left[\begin{array}{c}
\frac{-\sqrt{3}}{2} r \cos \phi-\frac{r}{2} \sin \phi \\
\frac{-\sqrt{3}}{2} r \sin \phi+\frac{r}{2} \cos \phi \\
0
\end{array}\right] \\
\mathbf{r}_{c}=\left[\begin{array}{c}
r_{c x} \\
r_{c y} \\
r_{c z}
\end{array}\right]=\left[\begin{array}{c}
r \sin \phi \\
-r \cos \phi \\
0
\end{array}\right]
\end{gathered}
$$

The parallel singularities of the manipulator occur when the determinant of matrix $\mathbf{A}$ is null. This condition is verified whenever the right lines on $U_{1} V_{1}, U_{2} V_{2}$ and $U_{3} V_{3}$ become parallel or converge in a common point. In the first case the platform loses capacity to apply force on the plane $X_{0}-Y_{0}$ in the normal direction to the parallel lines. In the second case the torsional rigidity of the platform vanishes with respect to the axis $Z_{0}$. Clearly, such phenomena are not suitable during the accomplishment of a task and consequently the manipulator should operate as far as possible from parallel singularities.

On the other hand, a serial singularity takes place when the determinant of matrix $\mathbf{B}$ is null. This situation is verified whenever the angle between links of a leg becomes $\pi$ (outstretched leg) or 0 (fully folded leg). In both cases the velocity of the extreme point $V_{i}(i \in[1,2,3])$ of the singular leg is null along the leg. Therefore, the platform cannot be displaced in arbitrary directions. Even if the mobility of the platform is degenerated under a serial singularity, the control on the active joints is preserved. Consequently, some strategies can be implemented in order to recover the mobility of the platform [36-37]. 


\subsection{Working modes}

The working modes $(W M)$ of a parallel manipulator can be identified by using the serial Jacobian matrix B. Accordingly, a $W M$ is a set of postures such that the elements on the main diagonal of $\mathbf{B}$ do not vanish and their signs do not change [11]. When a change of sign occurs for an element of the diagonal, then the $W M$ changes. It can be observed in Equation (3) that three elements are in the main diagonal of matrix $\mathbf{B}$ of the 3-RRR manipulator. Eight possible combinations can be obtained with signs of these elements, thus eight $W M$ are defined for this manipulator. Typical postures corresponding to the eight $W M$ of the 3-RRR manipulator can be appreciated in Figure 3.
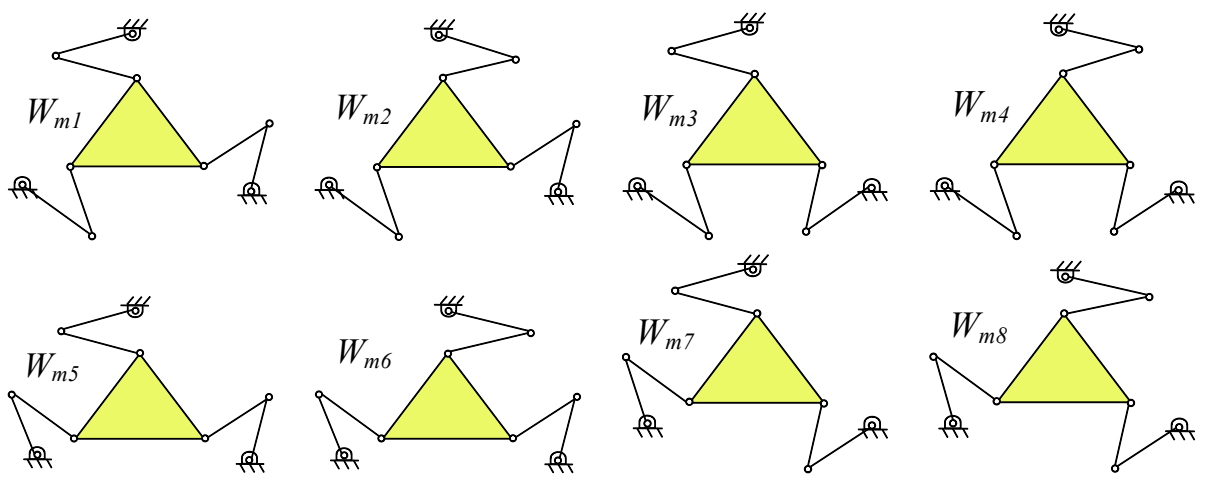

Fig. 3: Typical postures of the eight working modes associated to the 3-RRR parallel manipulator.

The different solutions of the inverse kinematics problem are grouped by the working modes. So, each $W M$ is associated to one solution of the inverse kinematic problem for a given pose of the platform. The posture that allows a change of a $W M$ has one leg in serial singularity. For instance in order to change from $W_{m 1}$ to $W_{m 2}$, it is observed in Figure 3 that the leg $O_{3} U_{3} V_{3}$ of the manipulator must pass through an outstretched (or fully folded) posture, which is singular.

\subsection{Inverse of the condition number}

The condition number $\kappa$ of a matrix $\mathbf{M}$ is defined as

$$
\kappa=\|\mathbf{M}\| \cdot\left\|\mathbf{M}^{-1}\right\|
$$

where $\|\mathbf{M}\|$ denotes any norm of $\mathbf{M}$. So, by employing the norm 2, the condition number is computed by

$$
\kappa=\frac{\sigma_{M}}{\sigma_{m}}
$$

where $\sigma_{M}$ and $\sigma_{m}$ are, respectively, the largest and the smallest singular values of M. Thus, $\kappa$ takes values greater or equal to 1. Because the units of the Jacobian matrix A are not homogenized, ordering the singular values does not make sense. Nevertheless, the matrix $\mathbf{A}$ homogenized, named $\overline{\mathbf{A}}$, can be obtained by dividing the third column of $\mathbf{A}$ by the characteristic length $L_{c}=\sqrt{2} r$, as proposed in [34]. Then, the inverse of the condition number of $\overline{\mathbf{A}}$ can be found as:

$$
\kappa^{-1}(\overline{\mathbf{A}})=\frac{\sigma_{m}}{\sigma_{M}}
$$


As shown in [34], using a geometric interpretation, the index $\kappa^{-1}(\overline{\mathbf{A}})$ behaves like a "distance" to parallel singularities. Note that $\kappa^{-1}(\overline{\mathbf{A}})$ is bounded by

$$
0 \leq \kappa^{-1}(\overline{\mathbf{A}}) \leq 1
$$

Now, $\kappa^{-1}(\overline{\mathbf{A}})$ can be employed as a performance index of the manipulator to avoid parallel singularities. Clearly, $\kappa^{-1}(\overline{\mathbf{A}})=0$ means that the manipulator is in a parallel singularity and $\kappa^{-1}(\overline{\mathbf{A}})=1$ means that the manipulator is on an ideal isotropic posture. In order to use postures as far as possible from parallel singularities when a task is being achieved, the index $\kappa^{-1}(\overline{\mathbf{A}})$ must be kept as large as possible.

\section{Feasibility maps}

Based in the notion introduced by Wenger et al. [38], a feasibility map for a serial manipulator with one degree of redundancy is a set of all the points $\left(\mathrm{t}, x_{m+1}\right)$ such that all the poses of the end effector associated to a desired task are reachable by using only non-singular postures without collisions and avoiding the joint limits. Here $m$ is the number of operational coordinates defining a pose of the end-effector, $x_{m+1}$ is an extra operational coordinate, suitably defined by the user, which depends on the joint variables, and $t$ is the execution time of the task. The set of points of a feasibility map is graphically represented as a 2D plot bounded by border curves corresponding to the singular postures of the manipulator, joint limits and collisions. This notion was applied in [39] to find feasible motions of a serial redundant manipulator for a complex task in a cluttered environment.

The usefulness of the feasibility maps was extended in [29] to solve the redundancy of parallel manipulators. In such a work the notion was improved by plotting the values of the index $\kappa^{-1}(\overline{\mathbf{A}})$ for the $3-\mathrm{RRR}$ manipulator following a path defined by the position coordinates $x_{p}$ and $y_{p}$ of the its moving platform. Since the orientation angle $\phi$ of the moving platform was not specified, it was chosen as the extra operational coordinate. As a result, the 3D obtained maps allow not only to observe the feasible orientations of the platform but also to identify those values of $\phi$ related to both the best and the worst postures of the manipulator. By definition, for a parallel manipulator the number of the feasibility maps is equal to the number of its working modes.

For instance, in the present paper the feasibility maps of the 3-RRR redundant manipulator is obtained for the closed path ACEGI of Figure 4, in which each right line segment is tracked in 8 seconds by the point $P$ of the platform using cycloidal motions. The map associated to $W_{m 1}$ is shown in Figure 5(a). Even if the feasibility maps are 3D plots, when a color code is used to designate different level of $\kappa^{-1}(\overline{\mathbf{A}})$ then these maps can be represented as planar plots that show the values of $\kappa^{-1}(\overline{\mathbf{A}})$ related to different orientations of the platform at each time step. The planar map in Figure 5(b) corresponds to the 3D map of Figure 5(a). The red color designates the largest values of $\kappa^{-1}(\overline{\mathbf{A}})$, and the blue dark color is associated to the smallest values of $\kappa^{-1}(\overline{\mathbf{A}})$ (those related to parallel singularities). The borders of the maps are defined by postures of the manipulator with one leg fully outstretched. So, the borders of the maps in magenta, yellow and green colors denote poses of the platform that define postures in serial singularities. Orientations of the platform outside the borders cannot be reached by the manipulator. The borders delimit the feasible values of $\phi$ for the desired path at each time step. In Figure 6 the feasibility maps related to the eight working modes of the manipulator are shown.

Each possible continuous trajectory inside the map, from the beginning up to the end of the task, that does not touch parallel singularities, represents a feasible motion of the manipulator which can be applied to achieve the task. If no such trajectories exist, then the task is not feasible. Hence, by a simple visual exploration of the feasibility map the user can recognize both feasible and non-feasible tasks. When the platform orientation is specified during motion, then the manipulator is non-redundant and the task is feasible if and only if one trajectory for $\phi$ can be identified in at least one feasibility map. However, if such an orientation is not specified, then the manipulator becomes kinematically redundant and an infinite number 
of trajectories of $\phi$ could be found. Thus, by considering an additional criterion to assess the quality of motion, one trajectory can be suitably determined in such a way that the performance of the manipulator be improved.

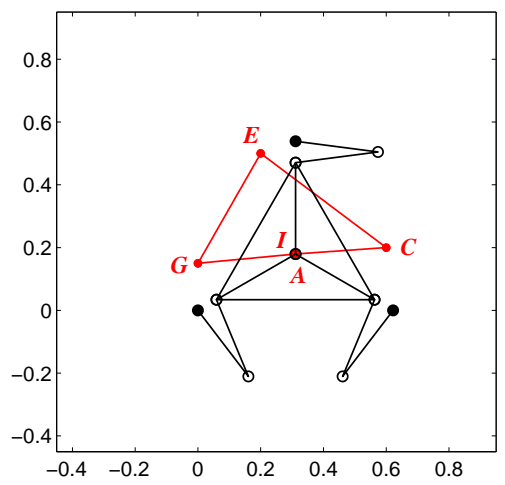

Fig. 4: Path to be accomplished by the manipulator in $32 \mathrm{sec}$.

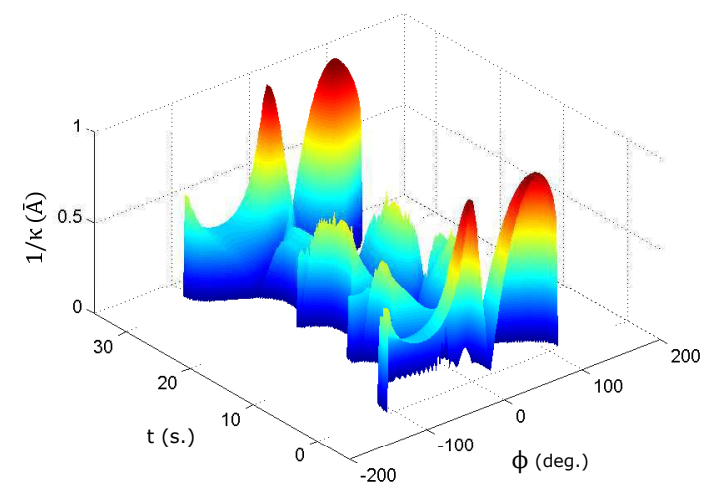

(a) Perspective view.

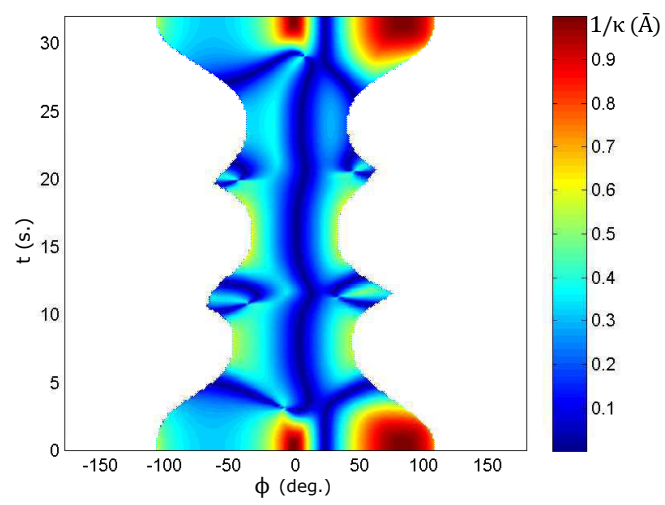

(b) Top view.

Fig. 5: Feasibility map associated to the $W_{m 1}$. Parallel singularities are characterized by $\kappa^{-1}(\overline{\mathbf{A}})=0$ and isotropic postures by $\kappa^{-1}(\overline{\mathbf{A}})=1$.

\section{Case study}

The improved method proposed in this paper for trajectory planning is presented in this Section. Without loss of generality the 3-RRR parallel manipulator is considered here for the task examined in Section 3.

The geometric parameters of the manipulator are: $l_{1}=0.26 \mathrm{~m}, l_{2}=0.26 \mathrm{~m}, r=0.29 \mathrm{~m}$ and $R=0.36 \mathrm{~m}$. These dimensions are those of the experimental prototype ITLag-IRCCyN shown in Figure 7. In this case, the centroid $P$ of the platform must follow the path shown in Figure 4. The path is a polygon composed of four right line segments. Each segment must be followed by a cycloidal motion of $P$ in a period of $8 \mathrm{~s}$. The coordinates of points $A, C, E, G, I$, referred to the frame $X_{0}-Y_{0}$ are $(0.311,0.18),(0.6,0.2),(0.2,0.5),(0$, $0.15),(0.311,0.18)$, respectively. The units are meters $(\mathrm{m})$. The initial and the final points of the polygonal path are $A$ and $I$, respectively, with the same coordinates. The feasibility maps associated to the eight working modes corresponding to the specified path are shown in Figure 6. 

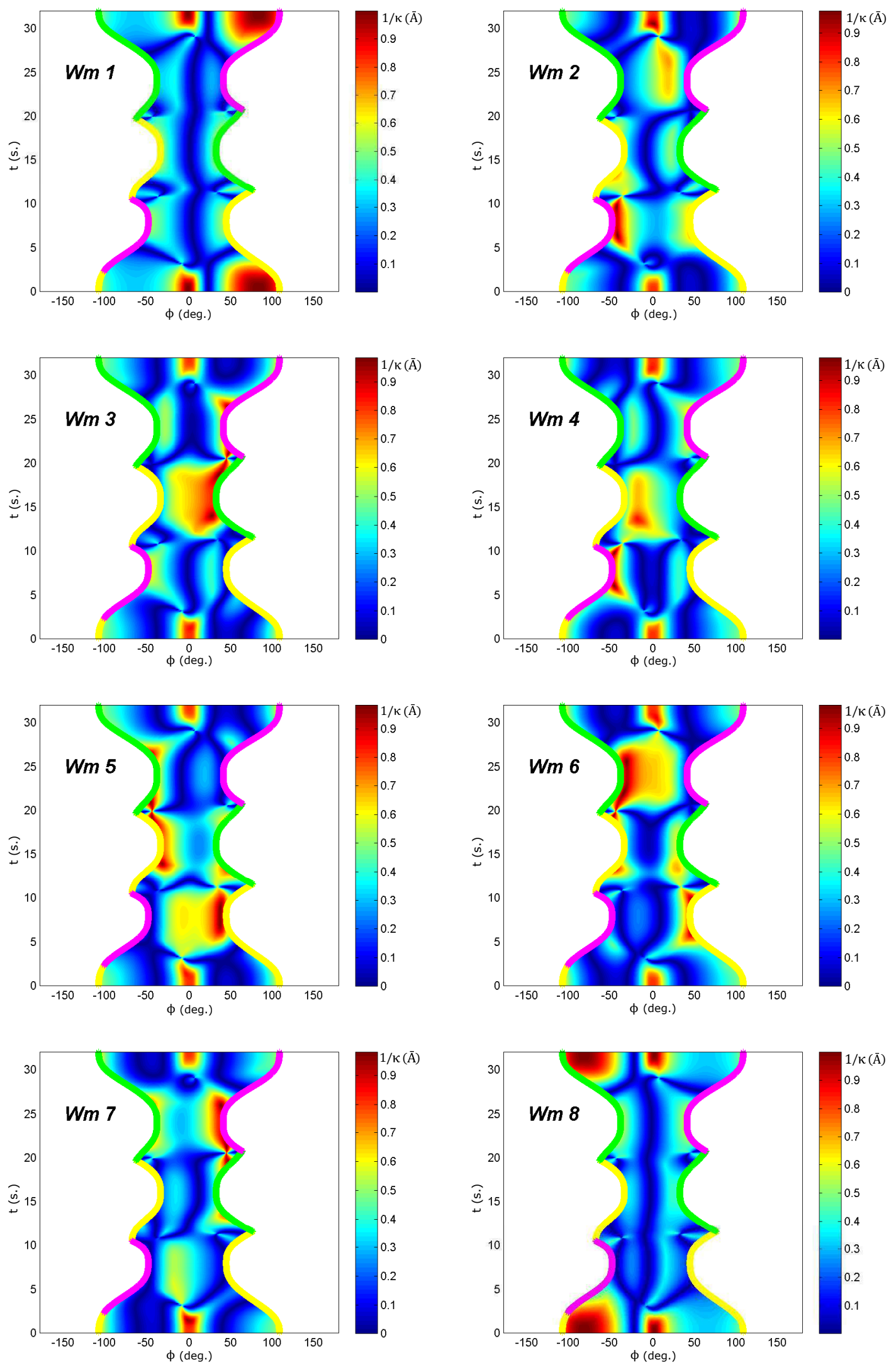

Fig. 6: Feasibility maps associated to the eight working modes of the 3-RRR manipulator for the path shown in Figure 4. 


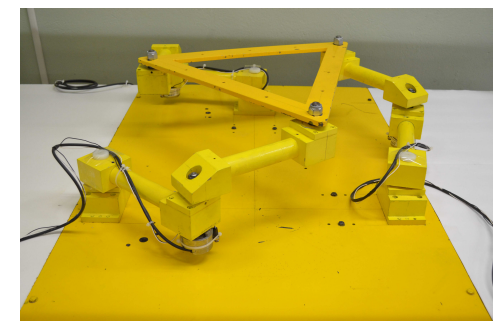

Fig. 7: 3-RRR Parallel robotic manipulator of ITLag-IRCCyN.

The proposed method for trajectory planning comprises three phases: preliminary exploration, establishment of a composed feasibility map and design of a trajectory inside this map. These phases are described in the following subsections.

\subsection{Preliminary exploration}

A preliminary exploration in maps of Figure 6 shows that no continuous trajectory, from $t=0$ sec up to $\mathrm{t}=32 \mathrm{sec}$, can be defined in a single map of a $W M$ without crossing a parallel singularity. Therefore, it is necessary to start and to finish the path in two distinct $W M$. To know the feasible changes of $W M$ and the legs that can be used to achieve these changes the matrix in Figure 8 must be applied. In this matrix different colors are associated to the legs: the yellow color for the leg 1, the green for the leg 2 and the magenta for the leg 3. On the other hand, the columns designate the $W M$ before the change (Starting $W M$ ) and the rows define the $W M$ after the change (Goal WM). For instance, the box at the intersection of the column 4 with the row 3 is filled with the magenta color. This means that the leg 3 can define a point in the common border of the feasibility maps associated to $W_{m 4}$ and $W_{m 3}$. Therefore a change of $W M$ can be completed from $W_{m 4}$ to $W_{m 3}$. It can be also observed that the box of column 4 with row 1 has no color, consequently no change is possible from $W_{m 4}$ to $W_{m 1}$.

By analyzing the maps in Figure 6 and taking into account the aforementioned matrix, it can be observed that some combinations of maps allow the manipulator to follow the whole desired path. Combinations such as $W_{m 2}-W_{m 1}-W_{m 3}$ or $W_{m 4}-W_{m 3}-W_{m 7}-W_{m 5}$ can be chosen to find a feasible trajectory.

When choosing one of these combinations, the user has to consider the starting point in the map corresponding to the starting $W M$, the transitional point to achieve the required change of $W M$, and the final point in the map of the goal WM. Additionally, the following recommendations need to be taken into account:

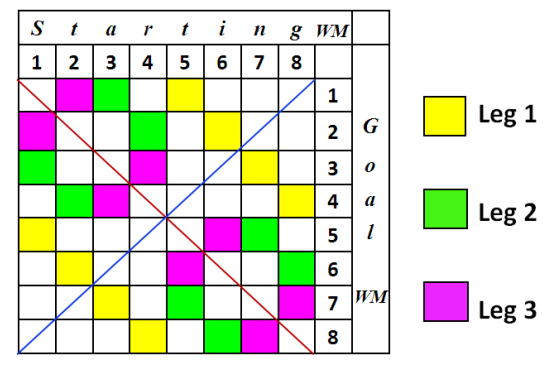

Fig. 8: Identification of legs associated to common borders of two $W M$ in feasibility maps.

a) To preserve a suitable kinetostatic performance of the manipulator, a candidate trajectory within a feasibility map must pass through platform orientations such that $\kappa^{-1}(\overline{\mathbf{A}})$ be as great as possible.

b) The candidate trajectory in a feasibility map cannot cross any border. 
In order to show how the three legs can be used to change the working modes in a task, the combination $W_{m 4}-W_{m 3}-W_{m 7}-W_{m 5}$ is chosen in this paper. However, in industrial applications other criteria could be employed to take a suitable combination.

It can be observed in Figure 9 that a path can be generated which crosses the maps associated to the chosen $W M$ without reaching zones of parallel singularities. The procedures to obtain both the composed map of Figure 9 and the path inside this map are described in the subsections 4.2 and 4.3.

\subsection{Completing a composed map}

The composed map of Figure 9 is obtained by cutting and joining at specific times the maps corresponding to the chosen working modes. A necessary condition to join two cut maps is that the slopes of the borders must be the same at the section to be joined. Specifically, the time derivatives of the borders must be zero on that section in order to make realistic the matching of the two trajectories inside the working modes to be joined. Otherwise, in the neighborhood of the matching point the trajectories could include points outside the feasibility maps, which would define unrealizable poses of the platform.

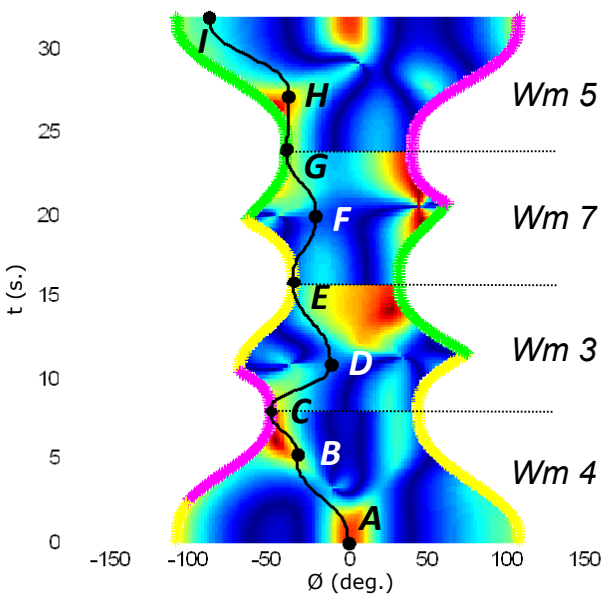

Fig. 9: Composed feasibility map associated to the chosen $W M$ and obtained trajectories.

The instant at which the time derivatives are zero on the borders of the maps is found by applying an optimization method for nonlinear functions [40]. It must be pointed out that, depending on the desired accuracy, the numerical process applied to find those points where the time derivatives vanish could require computing successive values of $\phi$ by using time steps smaller and smaller until convergence. Consequently, a procedure to computes $\phi$ on the border of the feasibility maps at arbitrary values of time must be established. This procedure is presented in the following paragraphs.

For an arbitrary instant $t$ during the tracking of the desired path of the platform, the coordinates $x_{p}, y_{p}$ of $P$ (Figure 1) are known. So, if the angle $\phi$ of the platform is obtained as function of these coordinates when a leg is outstretched, then such an angle will be implicitly known as function of time. Note that points on the border of the maps determine postures with one leg outstretched. Thus, the problem to be solved is to find the value of $\phi$ corresponding to the coordinates $x_{p}, y_{p}$ when a leg is outstretched. To solve this problem the postures in Figure 10 are considered.

In Figure 10 the following loop-closure equations are identified:

$$
\begin{aligned}
& \mathbf{r}_{7}=\mathbf{r}_{O_{1} V_{1}}+\mathbf{r}_{a} \\
& \mathbf{r}_{8}=\mathbf{r}_{O_{2} V_{2}}+\mathbf{r}_{b} \\
& \mathbf{r}_{9}=\mathbf{r}_{O_{3} V_{3}}+\mathbf{r}_{c}
\end{aligned}
$$



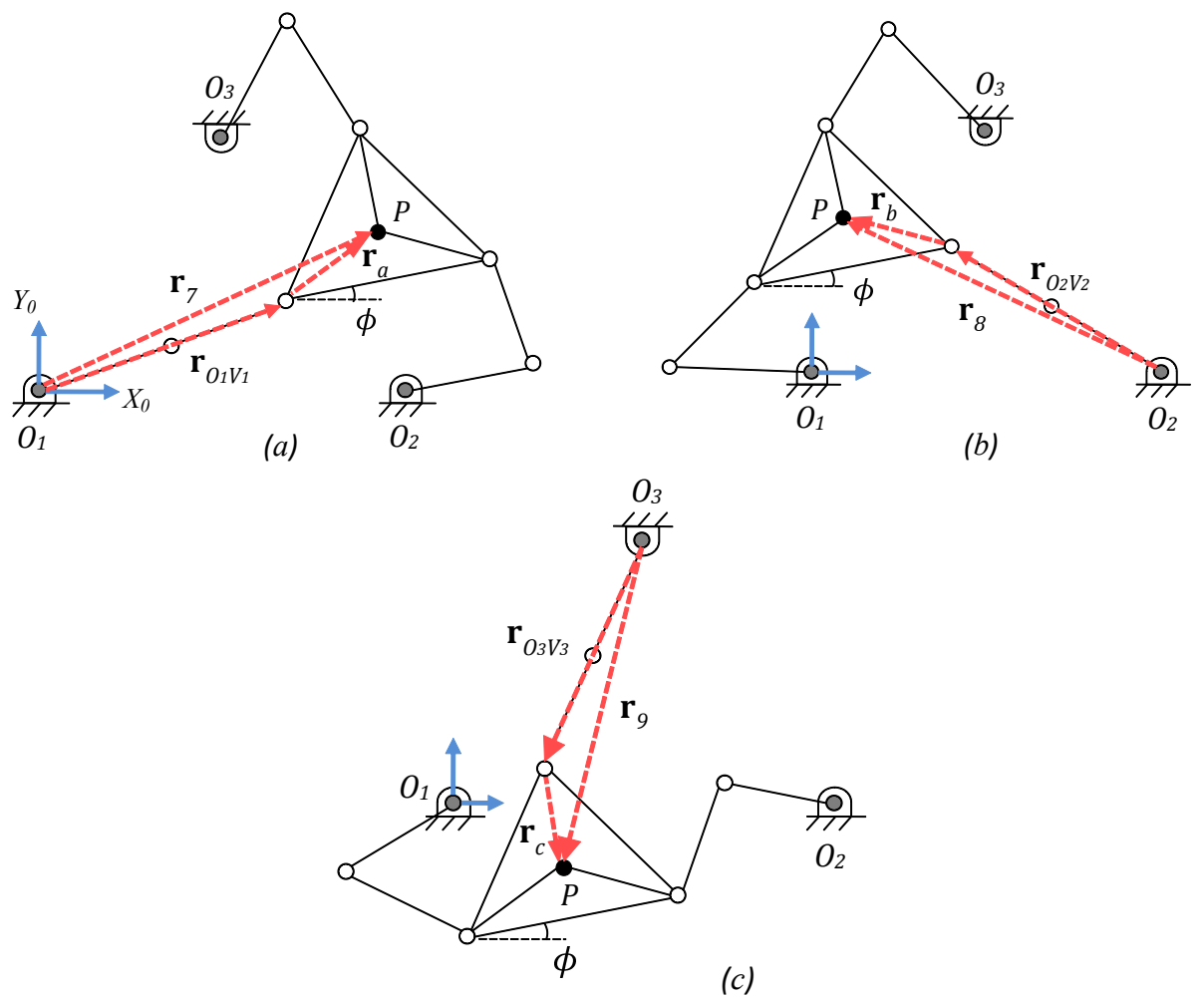

Fig. 10: Postures of the manipulator with legs outstretched. (a) Leg 1, (b) leg 2, (c) leg 3.

In these equations, for a specified position of $P$, the vectors $\mathbf{r}_{7}, \mathbf{r}_{8}$, and $\mathbf{r}_{9}$ are given in terms of their components in the frame $X_{0}-Y_{0}$. On the other hand, it is clear that $r=\left\|\mathbf{r}_{a}\right\|=\left\|\mathbf{r}_{b}\right\|=\left\|\mathbf{r}_{c}\right\|$, and $\left\|\mathbf{r}_{O_{i} V_{i}}\right\|=$ $l_{1}+l_{2}$ (for $i=1,2,3$ ), where the parameters $r, l_{1}$ and $l_{2}$ are known. So, the equations (13), (14) and (15) can be solved in order to find the Cartesian components of $\mathbf{r}_{a}, \mathbf{r}_{b}$ and $\mathbf{r}_{c}$ in terms of the coordinates $x_{p}, y_{p}$. Therefore, such components can be identified to those of the same vectors in equations (6), (7) and (8) given in terms of $\phi$, to obtain:

Leg1:

$$
\begin{aligned}
& \frac{\sqrt{3}}{2} r \cos \phi-\frac{r}{2} \sin \phi=\left(\varepsilon \cdot h_{1} r_{7 u y}+g_{1} r_{7 u x}\right) \\
& \frac{\sqrt{3}}{2} r \sin \phi+\frac{r}{2} \cos \phi=\left(g_{1} r_{7 u y}-\varepsilon \cdot h_{1} r_{7 u x}\right)
\end{aligned}
$$

$\underline{\operatorname{Leg} 2:}$

$$
\begin{aligned}
& \frac{-\sqrt{3}}{2} r \cos \phi-\frac{r}{2} \sin \phi=\left(\varepsilon \cdot h_{2} r_{8 u y}+g_{2} r_{8 u x}\right) \\
& \frac{-\sqrt{3}}{2} r \sin \phi+\frac{r}{2} \cos \phi=\left(g_{2} r_{8 u y}-\varepsilon \cdot h_{2} r_{8 u x}\right)
\end{aligned}
$$

Leg3:

$$
r \sin \phi=\left(\varepsilon \cdot h_{3} r_{9 u y}+g_{3} r_{9 u x}\right)
$$




$$
-r \cos \phi=\left(g_{3} r_{9 u y}-\varepsilon \cdot h_{3} r_{9 u x}\right)
$$

where:

$$
\begin{aligned}
& r_{7 u x}=\frac{x_{p}}{k_{1}}, \quad r_{7 u y}=\frac{y_{p}}{k_{1}}, \quad g_{1} \equiv \frac{r^{2}-\left(l_{1}+l_{2}\right)^{2}+k_{1}^{2}}{2 k_{1}}, \quad h_{1} \equiv \sqrt{r^{2}-g_{1}^{2}}, \quad g_{2} \equiv \frac{r^{2}-\left(l_{1}+l_{2}\right)^{2}+k_{2}^{2}}{2 k_{2}}, \quad h_{2} \equiv \sqrt{r^{2}-g_{2}^{2}}, \\
& r_{8 u x}=\frac{\left(x_{p}-S\right)}{k_{2}}, \quad r_{8 u y}=\frac{y_{p}}{k_{2}}, \quad g_{3} \equiv \frac{r^{2}-\left(l_{1}+l_{2}\right)^{2}+k_{3}^{2}}{2 k_{3}},
\end{aligned}
$$

in which:

$$
\begin{aligned}
& k_{1} \equiv \sqrt{x_{p}^{2}+y_{p}^{2}}, \quad k_{2} \equiv \sqrt{\left(x_{p}-S\right)^{2}+y_{p}^{2}}, \quad k_{3} \equiv \sqrt{\left(x_{p}-S / 2\right)^{2}+\left(y_{p}-\sqrt{3} S / 2\right)^{2}}, \\
& S \equiv \sqrt{3} R .
\end{aligned}
$$

Thus, for an arbitrary time t, the $\phi$ angle determined by one leg outstretched is computed by taking into account the current coordinates $\left(x_{p}, y_{p}\right)$ of $P$. This is achieved by solving the equations (16)-(21) for $\sin (\phi)$ and $\cos (\phi)$, and finally replacing their values in:

$$
\phi=\operatorname{atan} 2(\sin \phi, \cos \phi)
$$

By applying this process in the numerical method to find the points where the time derivatives vanish, the following coordinates are obtained for the transitional points $C, E$ and $G$ of feasibility maps in Figure 9:

$$
\begin{aligned}
& C\left(-49.09^{\circ}, \quad 8 s\right) \\
& E\left(-34.39^{\circ}, 16 s\right) \\
& G\left(-38.63^{\circ}, 24 s\right)
\end{aligned}
$$

Therefore, the feasibility maps of $W_{m 4}$ and $W_{m 3}$ are matched at $\mathrm{t}=8 \mathrm{~s}$, the maps of $W_{m 3}$ and $W_{m 7}$ are matched at $\mathrm{t}=16 \mathrm{~s}$, and finally the maps of $W_{m 7}$ and $W_{m 5}$ are matched at $\mathrm{t}=24 \mathrm{~s}$.

\subsection{Designing a trajectory}

To design the trajectory, some node points should be chosen inside the combined feasibility map of Figure 9. The nodes must be connected by curves that produce rotational cycloidal motions of the platform while the desired path is followed. Note that a cycloidal motion provides null velocities and accelerations at both the starting and the finishing points (the cycloidal motions have been extensively applied in cam design and the corresponding equations can be found in the literature, e.g. [41]). Thus, by observing the combined map, beside the transitional points $\mathrm{C}, \mathrm{E}$ and $\mathrm{G}$, the supplementary nodes A, B, D, F, H and I are chosen by the user. As pointed out in the previous subsection, the points $\mathrm{C}, \mathrm{E}$ and $\mathrm{G}$, are taken on the borders of the maps in order to achieve the required changes of working modes. On the other hand, the nodes A, B, D, F, $\mathrm{H}$ and $\mathrm{I}$ are judiciously chosen by the user in such a way that points of the connecting curves avoid parallel singularities.

The position of node A (starting point) in $W_{m 4}$ is chosen because a high value of the performance index is obtained on it, and a continuous curve can connect points A and C. However, it was observed that by using a single cycloidal motion between $\mathrm{A}$ and $\mathrm{C}$ the connecting curve touches parallel singularities. To avoid this 
problem, a supplementary node B is inserted between A and C; then the trajectory is kept out of singularities. Consequently, the rotation of the platform from $\mathrm{A}$ to $\mathrm{C}$ is completed by applying two successive cycloidal motions: one from A to B, and other from B to C. No one optimization is attempted. The supplementary node $\mathrm{B}$ is chosen by observation of the feasibility map.

Similarly, in order to avoid parallel singularities in the maps of the other working modes two cycloidal rotations of the platform are required to move from one transitional point of the trajectory to another. So, two cycloidal rotations are necessary for the segment C-E $\left(W_{m 3}\right)$, two for E-G $\left(W_{m 7}\right)$, and two for G-I $\left(W_{m 5}\right)$ in the map of Figure 9. The supplementary nodes inserted at each segment are the points D (in $W_{m 3}$ ), $\mathrm{F}\left(\right.$ in $W_{m 7}$ ) and $\mathrm{H}$ (in $W_{m 5}$ ). Their locations are defined in such a way that singularities are avoided by the connecting curves and reasonably high values of the index of performance are achieved. The smallest acceptable value of $\kappa^{-1}(\overline{\mathbf{A}})$ can be defined by the user depending on the complexity of the feasibility map. In this case study the lower bound of $\kappa^{-1}(\overline{\mathbf{A}})$ was arbitrarily defined as 0.1 . If values of this index are not greater than 0.1 by using only one supplementary node (like B, D, F and H) between the extreme points of a desired cycloidal motion, then more nodes could be incorporated in such a way that $\kappa^{-1}(\overline{\mathbf{A}})$ increases. On the other hand, nodes on the border (like C, E and G) cannot be changed because their time derivatives are zero and changes of working modes must be achieved on them. If the values of $\kappa^{-1}(\overline{\mathbf{A}})$ at such nodes were not acceptable, then other combinations of working modes could be explored.

The coordinates of the supplementary nodes A, B, D, F, H and I, chosen as described, are:

$$
\begin{aligned}
& A\left(0^{\circ}, \quad 0 s\right) \\
& B\left(-32.4^{\circ}, 5.28 s\right) \\
& D\left(-10.8^{\circ}, 10.88 s\right) \\
& F\left(-19.8^{\circ}, \quad 20 s\right) \\
& H\left(-37.8^{\circ}, 27.2 s\right) \\
& I\left(-88.2^{\circ}, \quad 32 s\right)
\end{aligned}
$$

The whole trajectory obtained in the combined map stabilizes the behavior of the angle $\phi$ of the platform to follow the desired path of $P$, with smooth changes of $W M$ and keeping the manipulator far away from parallel singularities. After obtaining the behavior of $\phi$, the inverse kinematic problems of position and velocity can be solved to find the corresponding active joint trajectories and histories of joint velocities. Such variables are plotted in Figure 11; they are used as control inputs for the actuators of the prototype in order to achieve the task. In Figure 12 a sequence of postures of the manipulator is shown during the execution of the task. A suitable behavior of the index $\kappa^{-1}(\overline{\mathbf{A}})$ during the task can be appreciated in Figure 13. The smallest value of this index was 0.19 at $t=19.6 \mathrm{sec}$, and the largest value was 0.81 at the beginning of the motion.

\section{Conclusion}

In trajectory planning of kinematically redundant parallel manipulators the joint trajectories to be applied must be found in order to achieve specified tasks by using suitable postures. Indeed, the parallel singularity avoidance is a necessary condition for feasible motions of parallel manipulators. The dexterous workspace of parallel manipulators decreases significantly because of such kinds of singularities. Thus, the accomplishment of continuous long paths becomes a complex issue. To solve this problem a method was proposed in this paper for kinematically redundant planar parallel manipulators that has to follow a specified path.

The method applies the feasibility maps associated to the $W M$ of the manipulator. Such maps allow the graphical assessment of the possible trajectories of the platform's orientation, which can be used during the accomplishment of the desired task. By analyzing the maps, the user might determine if one or several WM are needed.

The proposed method allows accomplishing the desired task even if several $W M$ are required to avoid parallel singularities. The trajectories in the maps use cycloidal rotational motions of the platform that produce smooth changes in orientations. When the manipulator arrives at (resp. departs from) a transitional 

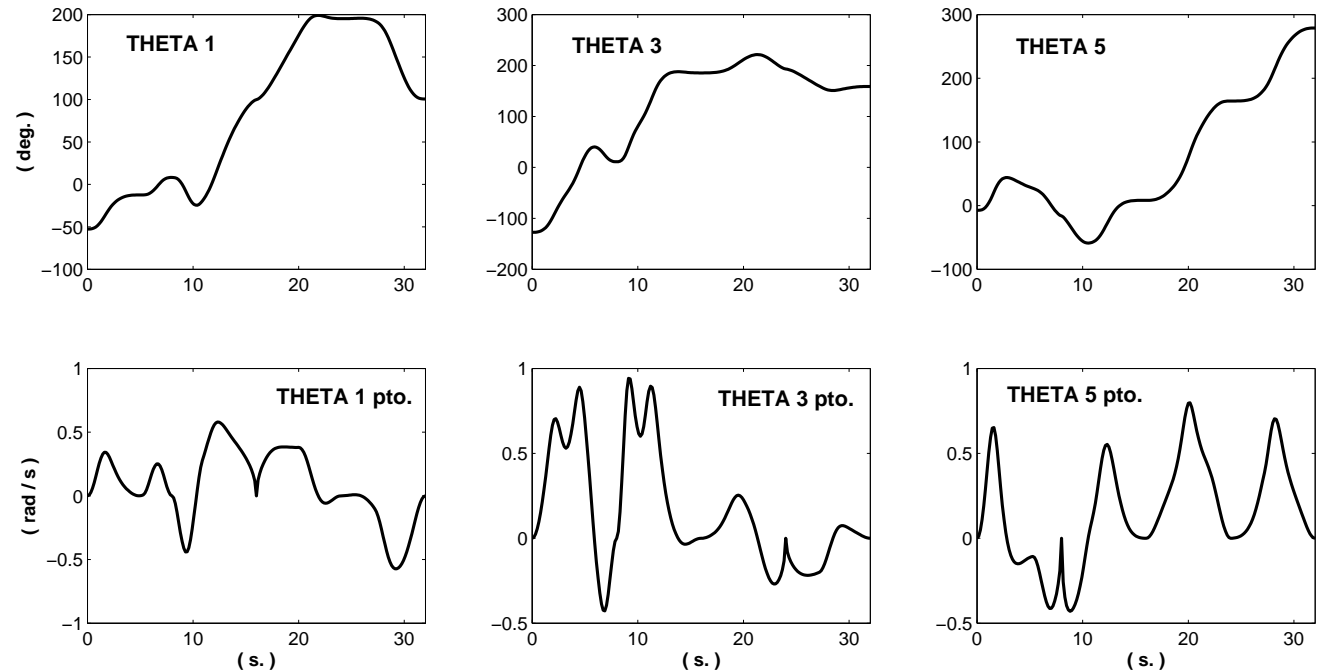

Fig. 11: Histories of the active joint variables and joint velocities.
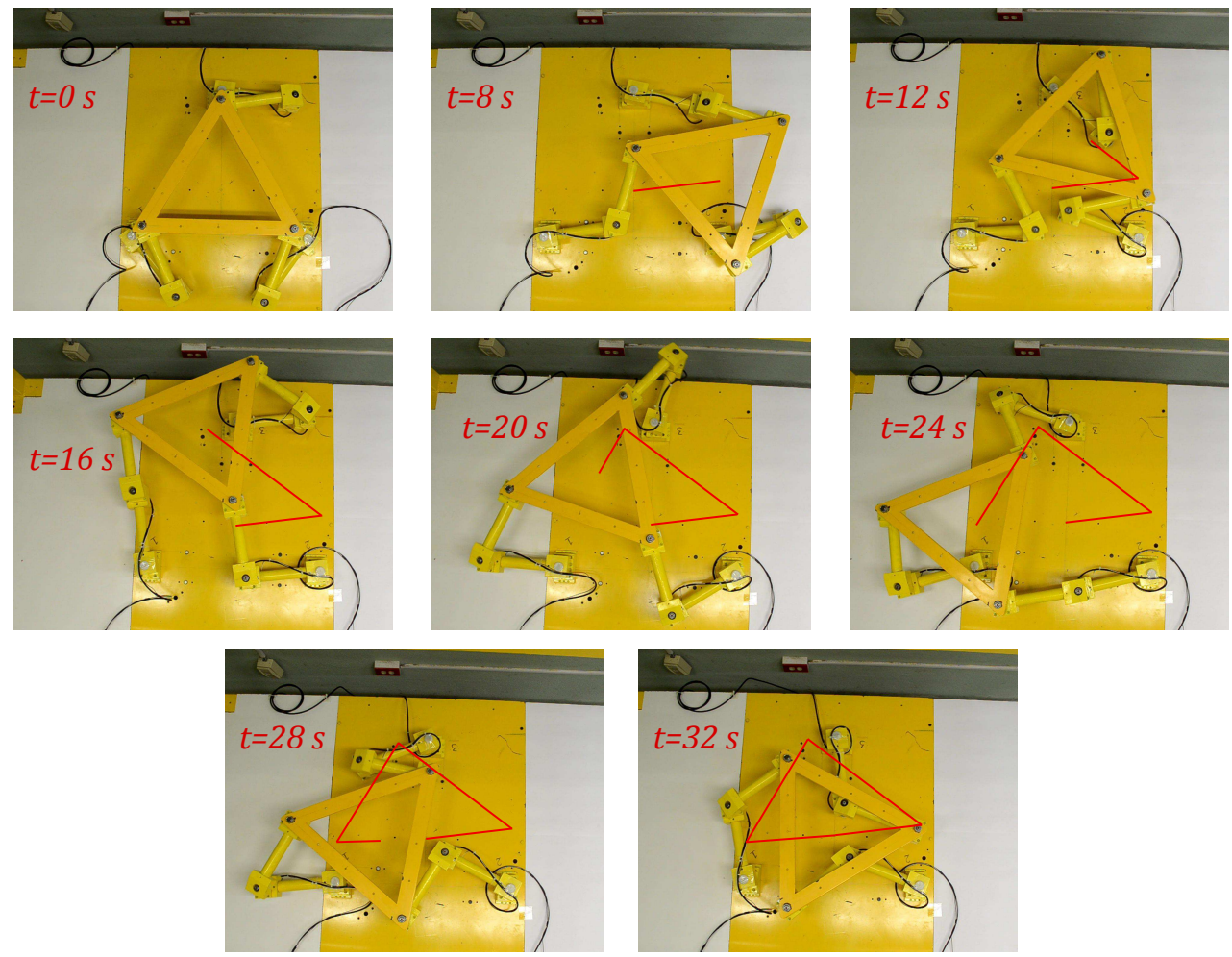

Fig. 12: Sequence of postures of the manipulator during the execution of the task. 


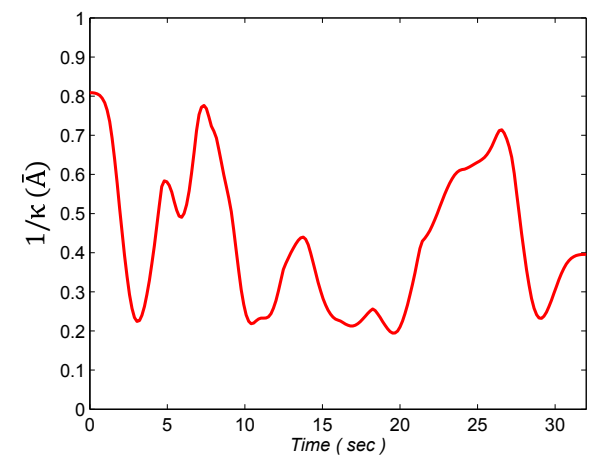

Fig. 13: Behavior of index $\kappa^{-1}(\overline{\mathbf{A}})$.

posture for a change of $W M$, particular conditions must hold in order to avoid large shaking forces in the manipulator. Indeed, on a transitional point of the map's border, both the arriving and the departing paths must be tangent to the border. This condition on the trajectories was not taken into account in [35].

The proposed method is general; it can be applied to redundant parallel manipulators with one degree of kinematic redundancy whose legs allow the required changing of working modes. In the case study of this paper a 3-RRR parallel manipulator was considered. The desired path was a polygon with four vertices; four working modes were needed to follow the whole path. The results of the trajectory planning process were applied to an experimental prototype, and the specified path was successfully completed with a suitable behavior of the manipulator. Smooth motions were observed when the desired path was followed even in transitional postures. Neither vibrations nor inappropriate shaking forces were perceived.

In the procedure presented in this paper the location of supplementary nodes of trajectories in the maps was achieved based on a visual analysis of the feasibility maps. Even if successful results were obtained, an automatic procedure for the location of such points could be suitable. To do that, some additional studies will be addressed in the future.

The index $K^{-1}(\overline{\mathbf{A}})$ employed in this paper for the 3-RRR manipulator is a dimensionally coherent measure of the "distance" of postures from singularities. Other kinetostatic performance indices could be applied in trajectory planning of parallel manipulators. For manipulators whose Jacobian matrix is dimensionally inhomogeneous there is no systematic way of defining a consistent index with a clear kinetostatic meaning. However, criteria based on the electric energy used by the actuators or on their maximal torques could be also interesting [42]. This kind of criteria will be considered in further works.

\section{Acknowledgments}

This work was supported by the CONACyT (National Council of Research and Technology) of Mexico and the CNRS (Centre National de la Recherche Scientifique) of France, under the project LAFMAA (Laboratoire Franco-Mexicain d'Automatique Appliquée), and also by the DGTN (General Direction of the National Institute of Technology) of the Ministry of the Public Education of Mexico.

\section{References}

[1] C.M. Gosselin, J. Angeles, Singularity analysis of closed-loop kinematic chains, IEEE Transactions on Robotics and Automation, 6, 281-290 (1990).

[2] S. Lahouar, S. Zeghloul, L. Romdhane, Singularity free path planning for parallel robots, Advances in Robot Kinematics, 235-242 (2008).

[3] E. Macho, O. Altuzarra, E. Amezua, A. Hernandez, Obtaining configuration space and singularity maps for parallel manipulators, Mechanism and Machine Theory, 44, 2110-2125 (2009)

[4] D. Chablat, $\mathrm{Ph}$. Wenger, The Kinematic analysis of a symmetrical three-Degree-Of-Freedom planar parallel manipulator, CISMIFToMM Symposium on Robot Design, Dynamics and Control, Kluwer Academic Publishers (2004). 
[5] F. Firmani, R.P. Podhorodeski, Singularity analysis of planar parallel manipulators based on forward kinematic solutions, Mechanism and Machine Theory, 44, 1386-1399 (2009).

[6] R. Di Gregorio, A novel method for the singularity analysis of planar mechanisms with more than one degree of freedom, Mechanism and Machine Theory, 44, 83-102 (2009).

[7] O. Bohigas, M. Henderson, L. Ros, J.M. Porta, A singularity-free path planner for closed-chain manipulators, IEEE International Conference on Robotics and Automation, 2128-2134, Saint Paul, MN, USA (2012).

[8] J-P. Merlet, Trajectory verification in the workspace for parallel manipulators, The International Journal of Robotics Research, 13, 326-333 (1994).

[9] B. Dasgupta, T.S. Mruthyunjaya, Singularity-free path planning for the Stewart plataform manipulator, Mechanism and Machine Theory, 33, 711-725 (1998).

[10] H. Abdellatif, B. Heimann, A novel multiple-heuristic approach for singularity-free motion planning of spatial parallel manipulators, Robotica, 26, 679-689 (2008).

[11] D. Chablat, Ph. Wenger, Working modes and aspects in fully parallel manipulators, IEEE International Conference on Robotics and Automation, 1964-1969, Leuven, Belgium (1998).

[12] D. Chablat, Ph. Wenger, Regions of feasible point-to-point trajectories in the Cartesian workspace of fully-parallel manipulators, ASME Design Engineering Technical Conferences, DETC99/DAC-8645 (1999).

[13] A. Figielski, I.A. Bonev, P. Bigras, Towards development of a 2-DOF planar parallel robot with optimal workspace use, IEEE International Conference on Systems, Man and Cybernetics, SMC 2007, Montreal, Canada (2007).

[14] L. Campos, F. Bourbonnais, I.A. Bonev, P. Bigras, Development of a five-bar parallel robot with large workspace, 2010 ASME International Design Engineering Technical Conferences \& Computers and Information in Engineering Conference, IDETC/CIE 2010, Montreal, Canada (2010).

[15] E. Macho, O. Altuzarra, Ch. Pinto, A. Hernandez, Workspaces associated to assembly modes of the 5R planar parallel manipulator, Robotica, 26, 395-403 (2008).

[16] D. Reveles, J.A. Pamanes, Planificación de rutas con cambio de modo de funcionamiento de un manipulador paralelo 2-RRR, XV COMRob: the AMROB Congress of Robotics, 111-116, Manzanillo, Mexico (2013).

[17] A. Liégeois, Automatic supervisory control of the configuration and behavior of multibody mechanisms, IEEE Transactions on Systems, Man and Cybernetics, SMC-7, 868-871 (1977).

[18] T. Yoshikawa, Analysis and control of robot manipulators with redundancy, Robotics Research: The First International Symposium, ed. M.Brady and R.Paul, MIT Press, 735-748 (1984).

[19] A.A. Maciejewski, C.A. Klein, Obstacle avoidance for kinematically redundant manipulators in dynamically varying environments, The International Journal of Robotics Research, 4, 109-117 (1985).

[20] Y. Nakamura, H. Hanafusa, Optimal redundancy control of robot manipulators, The International Journal of Robotics Research, 6, 32-42 (1987).

[21] S. Ma, D.N. Nenchev, Local torque minimization for redundant manipulators: a correct formulation, Robotica, 14, 235-239 (1996).

[22] J.A. Kuo, J. Sanger, Task planning for serial redundant manipulators, Robotica, 15, 75-83 (1997).

[23] J.A. Pamanes, L.A. Barron, C. Pinedo, Constrained optimization in redundancy resolution of robotic manipulators, 10th World Congress on Theory of Machines and Mechanisms, 1057-1066, Oulu, Finland (1999).

[24] H. Moreno, J.A. Pamanes, Ph. Wenger, D. Chablat, Global optimization of performance of a 2PRR parallel manipulator for cooperative tasks, Third International Conference on Informatics in Control, Automation and Robotics ICINCO, 516-522, Setubal, Portugal (2006)

[25] H. Hallak, Ph. Wenger, J.A. Pamanes, Multi-objective optimal kinematic inversion of a redundant 3-RRR planar parallel robot, 5th International Symposium on Robotics and Automation ISRA, 7-13, San Miguel Regla, Mexico (2006).

[26] O. Alba-Gomez, J.A. Pamanes, Ph. Wenger, Trajectory planning of a 3-RRR redundant parallel manipulator based on path planning of AGV's, VIII COMRob: the AMROB Congress of Robotics, 47-53, Mexico City, Mexico (2006).

[27] O. Alba-Gomez, J.A. Pamanes, Ph. Wenger, Trajectory planning of a 3-RRR redundant parallel manipulator in the presence of obstacles, 4th IEEE Latin America Robotic Symposium - IX COMRob: the AMROB Congress of Robotics, 126-133, Monterrey, Mexico (2007).

[28] O. Alba-Gomez, J.A. Pamanes, Ph. Wenger, Trajectory planning of parallel manipulators for global performance optimization, Advances in Robot Kinematics, Springer, 253-261 (2008).

[29] O. Alba-Gomez, Ph. Wenger, J.A. Pamanes, Consistent kinetostatic indices for planar 3-DOF parallel manipulators, application to the optimal kinematic inversion, 2005 ASME International Design Engineering Technical Conferences \& Computers and Information in Engineering Conference, DETC2005-84326, Long Beach, CA, USA (2005).

[30] S-H. Cha, T.A. Lasky, S.A. Velinsky, Kinematically-redundant variations of the 3-RRR mechanism and local optimization-based singularity avoidance, Mechanics Based Design of Structures and Machines: An International Journal, 35, 15-38 (2007).

[31] J. Aginaga, I. Zabalza, O. Altuzarra, J. Najera, Improving static stiffness of the 6-RUUS parallel manipulator using inverse singularities, Robotics and Computer-Integrated Manufacturing, 28, 458-471 (2012).

[32] J.A. Carretero, I. Ebrahimi, R. Boudreau, Overall motion planning for kinematically redundant parallel manipulators, ASME Journal of Mechanisms and Robotics, 4 (02), (2012).

[33] J. Angeles, Fundamental of Robotic Mechanical Systems, Springer-Verlag, New York, 1997.

[34] O. Alba-Gomez, Ph. Wenger, J.A. Pamanes, Performance indices for kinematically redundant parallel planar manipulators, Problems of Mechanics, 1, 1-24, (2006).

[35] O. Alba-Gomez, J.A. Pamanes, Ph. Wenger, Trajectory planning of a redundant parallel manipulator changing of working mode, 12th World Congress on Theory of Machines and Mechanisms, 1024-1029, Besançon, France (2007). 
[36] Ch. Chevallereau, Feasible trajectories in task space from a singularity for a non redundant or redundant robot manipulator, The International Journal of Robotics Research, 17, 56-69 (1998).

[37] J.A. Pamanes, E. Cuan, J.L. Zapata, An efficient approach for motion planning of non redundant manipulators using singular configurations, 2001 IASTED International Conference on Robotics and Manufacturing, 77-82, Cancun, Mexico (2001).

[38] Ph. Wenger, P. Chedmail, F. Reynier, A global analysis of following trajectories by redundant manipulators in the presence of obstacles, IEEE International Conference on Robotics and Automation, 3, 901-906, Atlanta, GA, USA (1993).

[39] J.A. Pamanes, Ph. Wenger, J.L. Zapata, Motion planning of redundant manipulators for specified trajectory tasks, Advances in Robot Kinematics, Kluwer Academic, 203-212 (2002).

[40] R.H. Byrd, J.C. Gilbert, J. Nocedal, A trust region method based on interior point techniques for nonlinear programming, Mathematical Programming, 89, 149-185 (2000).

[41] A.G. Erdman, G.N. Sandor, Mechanism Design: Analysis and Synthesis, Vol. 1, Prentice-Hall, Englewood Cliffs, 1984.

[42] R. Ur-Rehman, S. Caro, D. Chablat, Ph. Wenger, Multiobjective path placement optimization of parallel kinematics machines based on energy consumption, shaking forces and maximum actuators torques: application to the Orthoglide, Mechanism and Machine Theory, 45, 1125-1141 (2010). 